\title{
Retinal epithelitis as ocular side effects of oral biphosphonates
}

\author{
Giuseppe Lo Giudice, Valerio Crepaldi, Anton Giulio Catania* and Alessandro Galan \\ San Paolo Ophthalmic Center, San Antonio Hospital, Padova, Italy
}

\section{Case report}

Alendronate is a new bisphosphonate, primarily used for the treatment of osteoporosis and several other bone diseases. Herein we report a case of a patient showing an acute retinal pigment epithelitis (ARPE) following oral administration of alendronate due to osteoprosis. A 50-year old female referred to our observation complaining visual blurring and metamorphopsia in her left eye. She did not report ocular impairment in the past. Her clinical history was unremarkable for any systemic disease except she had being suffering for osteoporis for 6 months treated by means administration of oral alendronate. Her visual acuity was 20/40 OS and 20/20 OD respectively. There was no vitritis or anterior uveitis. At fundus examination subtle clumps of hyperpigmentation surrounded by a paler halo of hypopigmentation around macula of the retinal pigment epithelium (RPE) were shown in OS. In the right eye ophthalmoscopy showed some cluster of irregular pigmentation above the fovea associated with slight hypopigmentation surrounding the clump. Fluorescein angiography (FA) revealed an irregular patchy area of early hypofluorescence, surrounded by hyperfluorescence, while fading in the late phase (Figure 1A). Earlyphase and midphase indocyanine green angiography (ICG) showed a patchy macular hypofluorescence in both eyes (Figures 1B and 1C). Similar disseminated spots of hypofluorescence were shown at the periphery nasally to the optic disk; hypofluorescent halo with a petaloid like appearance of the macular area was observed in OS on late ICG. Patient discontinued treatment with alendronate. She underwent weekly ocular examination up to 4 weeks. Her symptoms improved in OS with VA of 20/20 in 1 week after discontinuation. FA and ICG revealed some patched areas of irregular hypofluorescence surrounding the old spots due to filling defect of choroidal vessels and characterized by late staining of dye on FA and no leakage (Figures 2A and 2B). Hypofluorescent areas were shown on both intermediate and late ICG phases with reducing size compared to the acute phases in the same areas.

Acute retinal pigment epithelitis (ARPE), also known as Krill's disease, is characterized by an acute and severe loss of vision in young, healthy adults with a self-limiting course and affecting both males and females equally [1]. It is thought to represent areas of RPE inflammation with breakdown of the pigment epithelial barrier and subsequent leakage of serous fluid. We report a case of a patient in which ARPE occurred after a long term administration of oral alendronate. These side effects are solved with discontinuation of therapy. A number of medications encompassing various forms of administration, including topical formulations, periocular and intraocular injections, and systemic medications, have been associated with uveitis. Bisphosphonates are primarily used to treat osteoporosis [2]. They are generally well tolerated, even though several medications in this class have been associated with uveitis or other ocular side effects [3]. Bisphosphonates have a particularly strong ocular toxicity including cases of orbital inflammation, scleritis, and uveitis and AMD [4]. In turn, bisphosphonates are believed to be proinflammatory. Specifically, they initiate an inflammatory cascade by activating $\mathrm{T}$ lymphocytes and upregulating systemic markers of inflammation, such as C-reactive protein, interleukin-6, and interleukin-8 [5]. In our report we consider ARPE as an adverse reaction to biphosphonate, even though a casual effect could not be ruled out. There was a time relation between both administration and discontinuation of the drug in absence of other drugs or chemicals or of concurrent disease that could explain the ocular side effect. A similar case has been reported, but conversely to our case symptoms begun early 1 month following intravenous infusion of biphosphonate (zoledronate). Also in our case a well-documented angiographic examination was carried out with both FA e ICG, showing the acute and subacute/chronic effects of ARPE secondary to oral biphosphonate administration. Drug-induced ocular side-effects is well-recognized and observed with a broad range of medications. A detailed drug history should be elicited, therefore, in all patients with otherwise unexplained symptoms. It is important that ocular symptomatology occurring in association with the use of novel medicines is diagnosed and data drawn to the attention of physicians, even though other reports are required to confirm such findings.

\section{Conflicts of interest}

All authors certify that they have NO affiliations with or involvement in any organization or entity with any financial interest, or non-financial interest in the subject matter or materials discussed in this manuscript. All authors were involved in design and conduction of study, collection, management, analysis, and interpretation of data, preparation, review and approval of manuscript.

Correspondence to: Anton Giulio Catania, San Paolo Ophthalmic Center, San Antonio Hospital, Via Jacopo Facciolati 71, Padova, 35127, Italy, Tel: +39 049 8216729; Fax: +39 049 2050153; E-mail: ag.catania@gmail.com

Received: October 04, 2017; Accepted: October 23, 2017; Published: October 25 2017 

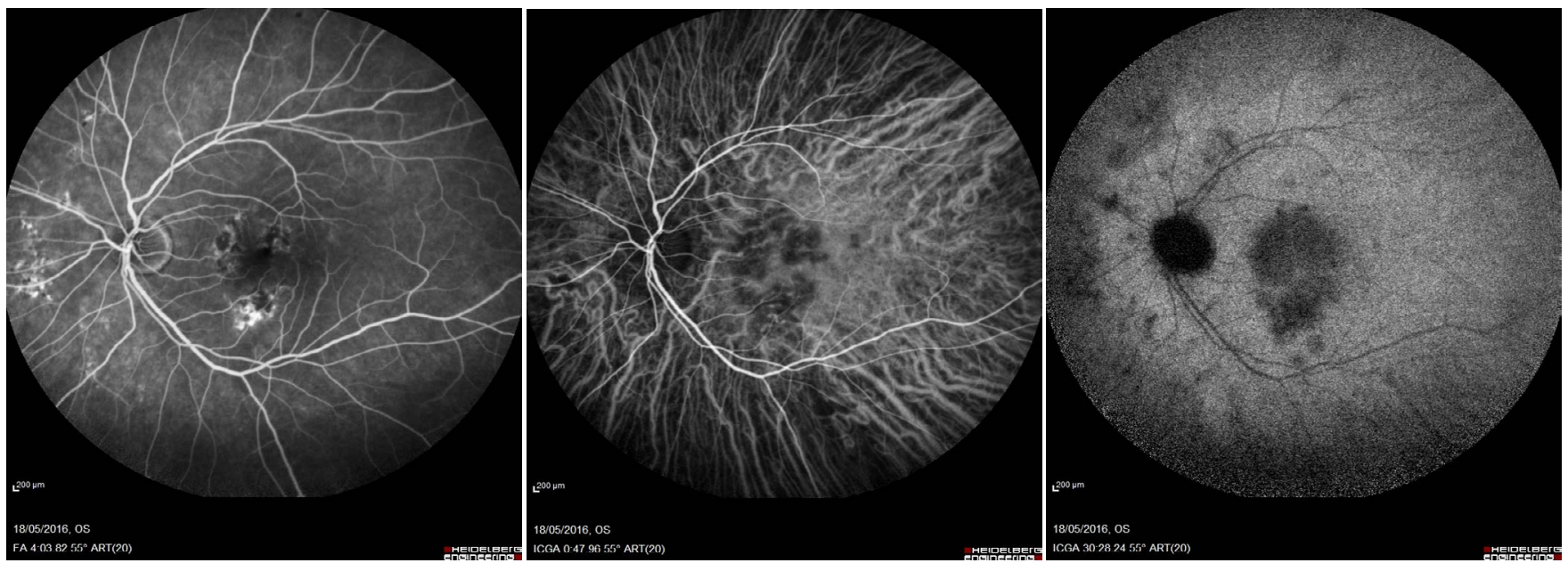

Figure 1. Fluorescein angiography showing early multiple spots of hypofluorescence surrounded by hyperfluorescent halo and located at the posterior pole in OS (A). Early and late phase of indocyanine green angiography (ICG) showing diffuse hypofluorescent dots in both eyes with multiple hypofluorescent spots nasal to the optic disk. An hypofluorescent halo with a petaloid-like appearence was also observed at the macular area in OS during the late ICG (B, C).
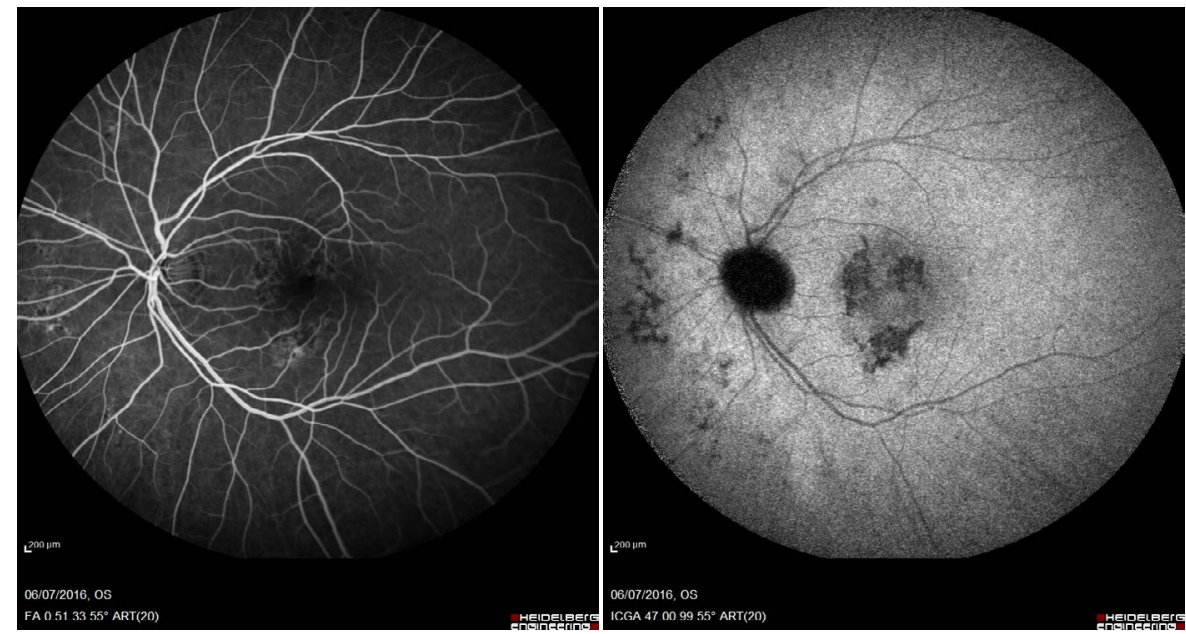

Figure 2. Fluorescein and indocyanine green angiography 4 weeks after drug discontinuation showing progressive resolution of active inflammatory foci at the level of retinal pigment epithelium, characterized by staining with no leakage during the late phases (A, B).

\section{References}

1. Krill AE, AF Deutman (1972) Acute retinal pigment epitheliitis. Am J Ophthalmol 74: 193-205.

2. Wysowski DK, Greene P (2013) Trends in osteoporosis treatment with oral and intravenous bisphosphonates in the United States, 2002-2012. Bone 57: 423-428. [Crossref]

3. Etminan M, Forooghian F, Maberley D (2012) Inflammatory ocular adverse events with the use of oral bisphosphonates: a retrospective cohort study. CMAJ 184: E431-E434. [Crossref]
4. Mammo Z, Guo M, Maberley D, Matsubara J, Etminan M (2016) Oral Bisphosphonates and Risk of Wet Age-Related Macular Degeneration. Am J Ophthalmol 168: 62-67. [Crossref]

5. Hewitt RE, Lissina A, Green AE, Slay ES, Price DA, et al. (2005) The bisphosphonate acute phase response: rapid and copious production of proinflammatory cytokines by peripheral blood gd T cells in response to aminobisphosphonates is inhibited by statins. Clin Exp Immunol 139: 101-111. [Crossref]

Copyright: (C2017 Giudice GL. This is an open-access article distributed under the terms of the Creative Commons Attribution License, which permits unrestricted use, distribution, and reproduction in any medium, provided the original author and source are credited. 